\title{
Erweiterung des Satzes, dass zwei polare Dreiecke perspectivisch liegen, auf eine beliebige Zahl von Dimensionen.
}

\author{
(Von Herrn Schläfli zu Bern.)
}

Im Quarterly mathematical Journal, vol. I, S. 191, S. 239 und S. 241 haben Salmon und Ferrers Beweise des im Titel ausgesprochenen Satzes für die Ebene und den Raum gegeben; in dem letzteren treten vier Reihen von je vier Coordinatenwerthen auf, die zusammen einen symmetrischen Determinant bilden. Da die diagonalen Elemente dieses Determinants in den dortigen Proportionen (S. 241 (1) bis (4)) nicht vorkommen, so sind sie variabel, aber durch drei Relationen, die aus der Aufgabe entspringen, mit einander verbunden. Ferrers zeigt dann, dass eine vierte von der Aufgabe verlangte Relation, die, wenn sie unabhängig wäre, alle vier Punkte vollständig bestimmte, nur eine nothwendige Folge der drei vorigen ist. Die Betrachtung des Ferrersschen Beweises hat mich überzeugt, dass er wesentlich auf diesem Satze beruht:

Das Verschwinden aller ersten Minore eines symmetrischen Determinants zählt nur für drei Bedingungen, während es für einen freien Determinant deren vier zählt.

Der Beweis dieses Satzes ist überflüssig, da er als specieller Fall in dem Kroneckerschen Satze enthalten ist, welchen Baltzer in seiner DeterminantenTheorie, $2^{\text {te }}$ Auflage S. 33 mittheilt.

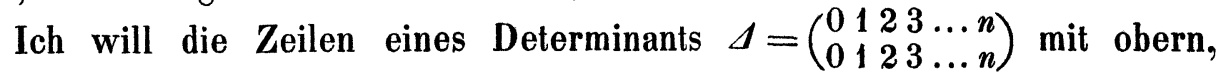
die Spalten mit untern Zeigern bezeichnen, und indem ich die Elemente selbst als Determinanten erster Ordnung betrachte, bezeichne ich diejenigen der obersten Zeile mit $\left(\begin{array}{l}0 \\ 0\end{array}\right),\left(\begin{array}{l}0 \\ 1\end{array}\right),\left(\begin{array}{l}0 \\ 2\end{array}\right), \ldots\left(\begin{array}{l}0 \\ n\end{array}\right)$, ferner setze ich $\left(\begin{array}{l}0 \\ 0\end{array}\right)\left(\begin{array}{l}1 \\ 1\end{array}\right)-\left(\begin{array}{l}0 \\ 1\end{array}\right)\left(\begin{array}{l}1 \\ 0\end{array}\right)$ $=\left(\begin{array}{l}01 \\ 01\end{array}\right)$, u. s. f.; das im Determinant mit $\left(\begin{array}{l}0 \\ 0\end{array}\right)$ multiplicirte Aggregat bezeichne

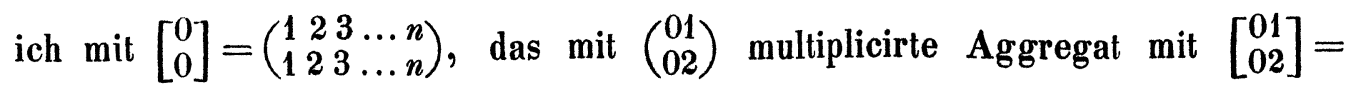
$\left(\begin{array}{llll}2 & 3 & 4 & \ldots . n \\ 3 & 1.4 & \ldots . n\end{array}\right)$, u. s. f., so dass z. B. $\left[\begin{array}{l}0 \\ 0\end{array}\right]\left[\begin{array}{l}r \\ 1\end{array}\right]-\left[\begin{array}{l}0 \\ 1\end{array}\right]\left[\begin{array}{l}r \\ 0\end{array}\right]=\left[\begin{array}{l}0 r \\ 01\end{array}\right] \Delta$ wird.

Dies vorausgesetzt, kann der Satz über die ersten Minore, um den es Journal für Mathematik Bd.LXV. Heft 3. 
sich hier handelt, folgendermassen ausgesprochen werden: es seien die zweiten Minore des Determinants $\Delta$ nicht sämmtlich gleich Null sondern mindestens einer derselben z. B. $\left[\begin{array}{l}01 \\ 01\end{array}\right]$ von Null verschieden, dann hat das Verschwinden der vier ersten Minore $\left[\begin{array}{l}0 \\ 0\end{array}\right],\left[\begin{array}{l}0 \\ 1\end{array}\right],\left[\begin{array}{l}1 \\ 0\end{array}\right],\left[\begin{array}{l}1 \\ 1\end{array}\right]$ das Verschwinden sämmtlicher ersten Minore zur Folge.

Im Allgemeinen reichen also die vier Bedingungen $\left[\begin{array}{l}0 \\ 0\end{array}\right]=0,\left[\begin{array}{l}0 \\ 1\end{array}\right]=0$, $\left[\begin{array}{l}1 \\ 0\end{array}\right]=0,\left[\begin{array}{l}1 \\ 1\end{array}\right]=0$ hin um das Verschwinden sämmtlicher ersten Minore des Determinants $\Delta$ zu bewirken. Wenn aber der Determinant symmetrisch ist, so ist $\left[\begin{array}{l}0 \\ 1\end{array}\right]=\left[\begin{array}{l}1 \\ 0\end{array}\right]$, und die Bedingungen sind bloss drei an Zahl.

Die Behandlung des allgemeinen Satzes, der Gegenstand dieses Aufsatzes ist, wird verständlicher werden, wenn ich zuerst den Ferrersschen Beweis für den räumlichen Fall mit stärkerer Hervorhebung dessen, was ich als Fundament betrachte, wiederhole.

Es sei $V=(w, x, y, z)^{2}$ das Polynom einer gegebenen Fläche zweiten Grades,

$$
\begin{aligned}
& p=k w+a x+b y+c z, \\
& q=a w+l x+h y+g z, \\
& r=b w+h x+m y+f z, \\
& s=c w+g x+f y+n z
\end{aligned}
$$

seien seine halben Abgeleiteten, also $V=p w+q x+r y+s z=0$ die Gleichung der Fläche. Dann sind die zwei Tetraeder $w x y z=0$ und pqrs $=0$ zu einander polar. Im Eck $\frac{\partial}{\partial w}$ des ersten Tetraeders ist $p=k, q=a, r=b$, $s=c$, im homologen Eck des zweiten Tetraeders $q=0, r=0, s=0$. Wenn also $\varphi$ eine Variable bedeutet und $p, q, r, s$ als Coordinaten gelten, die auf das zweite Tetraeder bezogen sind, so sind $\varphi, a, b, c$ Coordinaten des laufenden Punktes in der Geraden, die beide homologen Ecken verbindet. Wenn $\chi, \psi$, w ebenfalls Variable bedeuten, so sind alle vier Geraden, die je zwei homologe Ecken beider Tetraeder verbinden, durch die Zeilen der Matrix

$$
\left\{\begin{array}{cccc}
\varphi & a & b & c \\
a & \chi & h & g \\
b & h & \psi & f \\
c & g & f & \omega
\end{array}\right\}
$$


dargestellt. Verlangen wir nun, dass alle vier Punkte, je einer auf jeder Verbindungslinie, in einer Geraden liegen, so müssen sämmtliche ersten Minore dieser Matrix verschwinden. Da dieses nur drei Relationen liefert, denen die Variabeln $\varphi, \chi, \psi, \omega$ genügen müssen, so bleibt eine derselben frei, die verlangte Gerade wird nicht bestimmt, sondern kann sich einfach bewegen und beschreibt daher eine Fläche. zweiten Grades. Es sei $(p, q, r, s)$ ein Punkt der erzeugeñden Geraden, so steht es uns frei, für denselben die Bedingungen

$$
\left|\begin{array}{lll}
a & h & g \\
b & \psi & f \\
c & f & \omega
\end{array}\right|=0, \quad\left|\begin{array}{lll}
p & q & r \\
b & h & \psi \\
c & g & f
\end{array}\right|=0, \quad\left|\begin{array}{lll}
p & q & s \\
b & h & f \\
c & g & \omega
\end{array}\right|=0
$$

zu wählen und aus diesen $\psi, \omega$ zu eliminiren. Schliessen wir den Fall aus, wo $b g=c h$, diesem Systeme also entweder durch $h p=b q$ und eine Relation zwischen $\psi, \bullet \omega$, oder ohne eine Relation zwischen $p, q, r, s$ durch $c \psi=b f$, $b \omega=c f$ genügt würde, so ergiebt sich

$$
(b g-c h)(f p q+a r s)+(c h-a f)(g p r+b q s)+(a f-b g)(h p s+c q r)=0
$$

als Gleichung der Fläche zweiten Grades, die alle vier Verbindungsgeraden enthält, was man mit grösster Leichtigkeit verificiren kann.

Will man diese Fläche auf das erste Tetraeder beziehen, so braucht man nur $p, q, r, s$ durch $w, x, y, z$ und die Elemente $a, b, \ldots$ durch die entsprechenden Minore $A, B, \ldots$ zu ersetzen. Da $B G-C H=\Delta(b g-c h)$, etc., so erhält man $\Sigma(b g-c h)(F w x+A y z)=0$.

Wollte man diese Fläche in Bezug auf $V=0$ polarisiren, um diejenige Fläche zweiten Grades zu erhalten, die alle vier Geraden $(w=0, p=0)$, $(x=0, q=0),(y=0, r=0),(z=0, s=0)$ enthält, in denen je zwei homologe Seitenebenen beider Tetraeder sich schneiden, so hätte man, $b g-c h=\alpha$, $c h-a f=\beta, a f-b g=\gamma$ setzend, die Bedingungen

$$
\left\{\begin{aligned}
0 & =w p+x q+y r+z s, \\
t w & =\alpha f q+\beta g r+\gamma h s, \\
t x & =\alpha f p+\gamma c r+\beta b s, \\
t y & =\beta g p+\gamma c q+\alpha a s, \\
t z & =\gamma h p+\beta b q+\alpha a r
\end{aligned}\right.
$$

aus denen $p, q, r, s, t$ zu eliminiren wären. Der Determinant der Coefficienten in den vier untern Zeilen rechts ist $(\alpha \beta \gamma)^{2}$, und alle ersten Minore sind durch $\alpha \beta \gamma$ theilbar. Befreit man sie von diesem Factor, so erhält man 
in der obersten Zeile $2 a b c, a(b g+c h)$, etc. Wir wollen indess die Gleichung

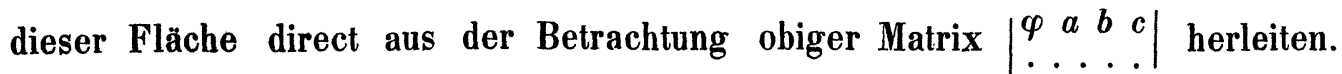
Wenn $\varphi$ willkürlich ist, so ist $\varphi w+a x+b y+c z=0$ die Gleichung irgend einer Ebene, die durch die Gerade $(w=0, p=0)$ gelegt ist, u. s. f. Denken wir uns nun die drei Relationen zwischen $\varphi, \chi, \psi, \omega$ erfüllt, vermöge deren sämmtliche ersten Minore jener Matrix verschwinden, so giebt es zwei von einander unabhängige Lösungen $(w, x, y, z),\left(w^{\prime}, x^{\prime}, y^{\prime}, z^{\prime}\right)$ des Systems aller vier Gleichungen $(\varphi w+a x+b y+c z=0$, etc. $)$. Dann ist aber auch der Punkt $\left(w+\lambda w^{\prime}, x+\lambda x^{\prime}, y+\lambda y^{\prime}, z+\lambda z^{\prime}\right)$ eine Lösung desselben Systems, und wir haben (wegen des willkürlichen Factors $\lambda$ ) eine Gerade, die allen vier Ebenen $\varphi w+a x+b y+c z=0$, etc. gemein ist und sich einfach bewegt, also eine Fläche zweiten Grades beschreibt, in der alle vier Durchschnitte $(w=0, p=0)$, etc. liegen. Um ihre Gleichung zu bekommen, brauchen wir nur $\psi$, $w$ aus den drei Gleichungen

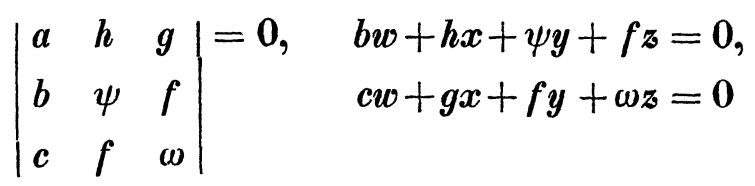

zu eliminiren und erhalten *)

$$
\begin{gathered}
T=a b c w^{2}+a h g x^{2}+b h f y^{2}+c g f z^{2}+(b g+c h)(a w x+f y z)+(c h+a f)(b w y+g x z) \\
+(a f+b g)(c w z+h x y)=0 .
\end{gathered}
$$

Dass diese Fläche durch die vier Geraden $(w=0, p=0)$, etc. geht, verificirt sich sogleich an der Form

$T=a b c w^{2}+w(a(b g+c h) x+b(c h+a f) y+c(a f+b g) z)+(a x+b y+c z)(g h x+h f y+f g z)$

ihres Polynoms, wobei zu beachten ist, dass die zweite Gerade $g h x+h f y+f g z$ $=0$, in der die Ebene $w=0$ von der Fläche $T=0$ geschnitten wird, die Lösung unserer Aufgabe in der Ebene für das Dreieck $x y z=0$ und sein polares in Bezug auf die Curve $l x^{2}+m y^{2}+n z^{2}+2 f y z+2 g x z+2 h x y=0$ darstellt. Der Discriminant von $2 T$ ist $(\alpha \beta \gamma)^{2}$, und seine Minore sind $\alpha \beta \gamma \times$ $(0, \alpha f, \beta g, \gamma h)$, etc.

Wir gehen nun an die allgemeine Aufgabe. Es sei

$$
V=a_{0} x_{0}^{2}+a_{1} x_{1}^{2}+\cdots+a_{n} x_{n}^{2}+2\left(\begin{array}{l}
0 \\
1
\end{array}\right) x_{1} x_{1}+2\left(\begin{array}{l}
0 \\
2
\end{array}\right) x_{0} x_{2}+\cdots+2\left(\begin{array}{c}
n-1 \\
n
\end{array}\right) x_{n-1} x_{n}=0
$$

*) Vergleiche Quart. Journal I., p. 195 unten. 
die quadratische Gleichung, in Bezug auf die das Gebilde $x_{0} x_{1} x_{2} \ldots x_{n}=0$ polarisirt werden soll (es ist $\left(\begin{array}{l}1 \\ 0\end{array}\right) \doteq\left(\begin{array}{l}0 \\ 1\end{array}\right)$, etc. angenommen). Wird

$$
p_{0}=a_{0} x_{0}+\left(\begin{array}{l}
0 \\
1
\end{array}\right) x_{1}+\left(\begin{array}{l}
0 \\
2
\end{array}\right) x_{2}+\cdots+\left(\begin{array}{l}
0 \\
n
\end{array}\right) x_{n}, \quad \text { etc. }
$$

gesetzt, so ist das zweite zum vorigen polare Gebilde $p_{0} p_{1} p_{2} \ldots p_{n}=0$. Da der erste Theil der Aufgabe [die homologen Ecken beider polaren Gebilde durch Gerade zu verbinden, auf jeder von $n-2$ dieser Geraden je einen Punkt willkürlich zu wählen, durch diese $n-2$ Punkte eine einfach drehbare lineare Gleichung (im Raume wäre es ein Ebenenbüschel) so zu legen, dass sie noch mit der $(n-1)^{\text {ten }}$ Verbindungslinie einen Punkt und mit der $n^{\text {ten }}$ einen Punkt gemein habe, worauf sie von selbst mit der $(n+1)^{\text {ten }}$ Verbindungslinie einen Punkt gemein haben wird, endlich alfe jene erstgenannten $n-2$ Punkte unabhängig von einander auf den betreffenden Verbindungslinien zu bewegen, die drehbare lineare Gleichung, welche $n+1$ Punkte mit sämmtlichen Verbindungslinien (mit jeder einen) gemein hat, zu drehen und nun die höhere Gleichung zu finden, die von der $(n-1)$ fach beweglichen linearen Gleichung umhiullt wird] zu grossen Schwierigkeiten unterliegt, so wenden wir uns sogleich zum zweiten Theile, eine Gerade zu ziehen, die mit jedem der Durchschnitte $\left(x_{r}=0, p_{r}=0\right),(r=0,1,2, \ldots n)$ einen Punkt gemein hat. Statt der gegebenen $a_{0}, a_{1}, \ldots a_{n}$ führen wir als diagonale Elemente der Matrix

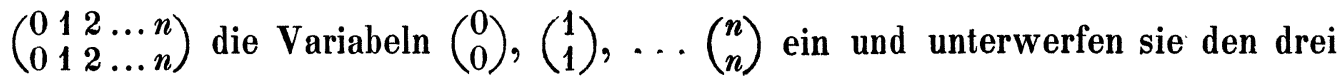
Bedingungen, die das Verschwinden aller ersten Minore bewirkt. Dann werden alle $n+1$ Gleichungen $\left(\begin{array}{l}r \\ 0\end{array}\right) x_{1}+\left(\begin{array}{l}r \\ 1\end{array}\right) x_{1}+\left(\begin{array}{l}r \\ 2\end{array}\right) x_{2}+\cdots+\left(\begin{array}{l}r \\ n\end{array}\right) x_{n}=0,(r=0,1, \ldots n)$ durch zwei von einander unabhängige Coordinatengruppen befriedigt; ihre Lösungen bilden also eine Gerade; und da $n-2$ unabhängige Variabeln, z. B. $\left(\begin{array}{l}3 \\ 3\end{array}\right),\left(\begin{array}{l}4 \\ 4\end{array}\right), \ldots\left(\begin{array}{l}n \\ n\end{array}\right)$ übrig bleiben, so beschreibt die Gerade ein krummes Continuum von $n-1$ Dimensionen, das also durch eine einzige Gleichung $T=0$ auszudrücken ist. Nimmt man z. B. aus den linearen Gleichungen mit $r=2,3, \ldots n$ die Werthe für $\left(\begin{array}{l}2 \\ 2\end{array}\right),\left(\begin{array}{l}3 \\ 3\end{array}\right), \ldots\left(\begin{array}{l}n \\ n\end{array}\right)$ und substituirt sie in der Bedingung $-\left[\begin{array}{l}0 \\ 1\end{array}\right]=0$, so erhält man eine Gleichung $(n-1)^{\text {ten }}$ Grades in $x_{0}, x_{1}$, $x_{2}, \ldots x_{n}$. Denn man muss z. B. die Zeilen $2,3, \ldots n$ resp. mit $-x_{2}$, $-x_{3}, \ldots-x_{n}$ multipliciren, um substituiren zu können. Es sei $T=$ $(-1)^{n} x_{2} x_{3} \ldots x_{n}\left[\begin{array}{l}0 \\ 1\end{array}\right]$, wenn die Substitutionen ausgeführt sind. Giebt man der 


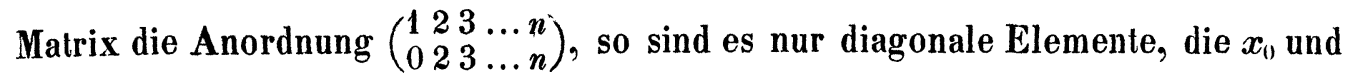
$x_{1}$ enthalten. Daher ist in $\boldsymbol{T}$ der Coefficient von $x_{0}^{n-1}$ gleich $\left(\begin{array}{l}0 \\ 1\end{array}\right)\left(\begin{array}{l}0 \\ 2\end{array}\right)\left(\begin{array}{l}0 \\ 3\end{array}\right) \ldots\left(\begin{array}{l}0 \\ n\end{array}\right)$, und das Aggregat aller Terme, in denen $x_{2}, x_{3}, \ldots x_{n}$ fehlen, kann durch

$$
\left(\begin{array}{l}
0 \\
1
\end{array}\right)\left(\left(\begin{array}{l}
0 \\
2
\end{array}\right) x_{0}+\left(\begin{array}{l}
1 \\
2
\end{array}\right) x_{1}\right)\left(\left(\begin{array}{l}
0 \\
3
\end{array}\right) x_{0}+\left(\begin{array}{l}
1 \\
3
\end{array}\right) x_{1}\right) \ldots\left(\left(\begin{array}{l}
0 \\
n
\end{array}\right) x_{0}+\left(\begin{array}{l}
1 \\
n
\end{array}\right) x_{1}\right)
$$

därgestellt werden, woraus sogleich auch die Gestalt der Coefficienten von $x_{1}^{n-1-\lambda} x_{1}^{\lambda}$ erhellt. Aber die Coefficienten der Terme, welche $x_{2}, x_{3}, \ldots$ enthalten, aus der Matrix von $-\left[\begin{array}{l}0 \\ 1\end{array}\right]$ abzuleiten, wird mir zu schwierig. Ich will daher zunächst nur zeigen, dass man die Aufgabe von $n$ Dimensionen auf $n-1$ zurück bringen kann. Wenn in dieser Matrix die Zeilen $2,3, \ldots$ $n$ wie oben gesagt multiplicirt sind, so dürfen wir die Zeile 2 dadurch verändern, dass wir zu ihr alle folgenden Zeilen und das $x_{1}$ fache der Zeile 1 addiren. Dadurch wird

$$
\begin{aligned}
T=x_{0}\left\{(-1)^{n} x_{3} x_{4} \ldots\right. & \left.x_{n}\left(\left[\begin{array}{l}
1 \\
2
\end{array}\right]-\left(\begin{array}{l}
0 \\
0
\end{array}\right)\left[\begin{array}{l}
01 \\
02
\end{array}\right]\right)\right\}+\left(\left(\begin{array}{l}
0 \\
1
\end{array}\right) x_{1}+\left(\begin{array}{l}
0 \\
2
\end{array}\right) x_{2}+\cdots+\left(\begin{array}{l}
0 \\
n
\end{array}\right) x_{n}\right) \\
& <(-1)^{n-1} x_{3} x_{4} \ldots x_{n}\left[\begin{array}{l}
01 \\
02
\end{array}\right] .
\end{aligned}
$$

Für $x_{0}=0$ reducirt sich also dieser Ausdruck auf seinen zweiten Term, und dieser zerfällt in zwei Factoren, von denen der erste lineare unserer Aufgabe entspricht, der zweite $(n-2)^{\text {ten }}$ Grades aber, wenn darin auch $x_{v}=0$ gesetzt wird, in Bezug auf die Matrix $\left(\begin{array}{llll}1 & 2 & 3 & \ldots \\ 1 & 2 & 3 & \ldots\end{array}\right)$ gerade dieselbe Function ist, welche $T$ in Bezug auf $\left(\begin{array}{lllll}0 & 1 & 2 & 3 & \ldots \\ 0 & 1 & 2 & 3 & \ldots\end{array}\right)$ war. Da in $T$ kein Term alle Coordinaten zugleich enthalten kann, sondern immer mindestens zwei fehlen müssen, so ist hiemit die Möglichkeit gezeigt, die Coefficienten successiv durch Aufsteigen von $3 \mathrm{zu} \mathrm{4}$, von $4 \mathrm{zu} 5$, .. Dimensionen und jedesmalige Multiplication zu berechnen. Man kann aber auch das Gesetz errathen, nach dem der Coefficient irgend eines Terms in $\boldsymbol{T}$ gebildet ist, und zeigen, dass die so definirte Function für jede Lösung von $\left(x_{0}=0, p_{0}=0\right)$, etc. verschwindet. Wenn dann ferner gezeigt wird, dass diese Bedingungen mehr als hinreichen, um die Coefficienten in einer Function $\left(x_{0}, x_{1}, x_{2}, \ldots x_{n}\right)^{n-1}$ zu bestimmen, so ist damit auch die Identität der definirten Function mit $T$ bewiesen.

Eine Function $\left(x_{0} \ldots x_{n}\right)^{n-1}$ zählt $\left(\begin{array}{c}2 n-1 \\ n-1\end{array}\right)-1$ Elemente, wenn es nur auf die Verhältnisse der Coefficienten ankommt: Ihr Continuum würde von $\left(x_{0}=0, p_{1}=0\right)$ in einem krummen Continuum $(n-1)^{\text {ten }}$ Grades mit $n-1$ ho- 
mogenen Variabeln geschnitten, das durch $\left(\begin{array}{c}2 n-3 \\ n-2\end{array}\right)-1$ Punkte bestimmt würde. Damit also jenes Continuum das lineare Continuum $\left(x_{0}=0, p_{10}=0\right)$ nicht schneiden könne, sondern es ganz in sich enthalte, müssen jenem in diesem $\left(\begin{array}{c}2 n-3 \\ n-2\end{array}\right)$ Punkte gegeben werden. Da dieses sich $n+1$ Male wiederholt, so sind dem gesuchten krummen Continuum $(n+1)\left(\begin{array}{c}2 n-3 \\ n-2\end{array}\right)$ Punkte gegeben. Der Ueberschuss dieser Zahl über die Anzahl der Elemente der gesuchten Function beträgt $(n-1)\left(\begin{array}{c}2 n-3 \\ n-3\end{array}\right)+1$, also für $n=2,3,4,5$ resp. 1, 3, 16, 85. Die Function $T$ ist also durch die erwähnten Bedingungen mehr als bestimmt.

Wenn den untern Zeigern 1, 2, 3, ..n irgend eine Anordnung eben so vieler zum Theil wiederholter Zeiger, 'unter denen aber der fremde Zeiger 0 sich nicht befindet, übergesetzt wird, so ist es nicht möglich, Kreisläufe wie $\left(\begin{array}{l}1 \\ 1\end{array}\right),\left(\begin{array}{l}1 \\ 2\end{array}\right)\left(\begin{array}{l}2 \\ 1\end{array}\right),\left(\begin{array}{l}1 \\ 2\end{array}\right)\left(\begin{array}{l}2 \\ 3\end{array}\right)\left(\begin{array}{l}3 \\ 1\end{array}\right), \ldots$ zu vermeiden. Denn fängt man mit einem Element an, dessen unterer Zeiger auch oben irgendwo vorkommt, so kann man eine Kette von Elementen bilden, wo jeder obere Zeiger eines Elements mit dem untern des nachfolgenden übereinstimmt, und diese Kette könnte nur dann offen bleiben, wenn sie mit einem obern Zeiger schlösse, der unten nicht vorkäme. Da dieses nicht der Fall ist, so muss die Kette einmal sich schliessen, d. h. der obere Zeiger des letzten Elements muss mit dem untern Zeiger dieses oder irgend eines der vorangegangenen Elemente übereinstimmen; und die Combination von Elementen enthält mindestens einen Kreislauf. Ich brauche hierfür nur an die Abhandlungen von Cauchy (Journal de l'école polytechnique Cah. 17, p. 37) und von Jacobi (dieses Journal Bd. 22, S. 288) zu erinnern. Ich will nun eine Function $(T)$ definiren, deren Identität mit $\boldsymbol{T}$ zu untersuchen ist.

Um den Coefficienten von $x_{10}^{\alpha} x_{1}^{\beta} x_{2}^{\gamma} x_{3}^{\delta} x_{4}^{\varepsilon},(\alpha+\beta+\gamma+\delta+\varepsilon=n-1)$, in $(T) z u$ erhalten, setze man der festen Reihe $1234 \ldots n$ unterer Zeiger alle diejenigen Permutationen von $0^{\alpha+1} 1^{\beta} 2^{\gamma} 3^{\delta} 4^{\varepsilon}$ über, welche keine Kreisläufe erzeugen, und fasse jede Anordnung als Product derjenigen Elemente von $V$ auf, welche durch je zuei übereinanderstehende Zeiger bezeichnet sind. Die Summe aller solchen Producte, deren Anzahl gleich der Permutationszahl der Coordinatencombination ist, ist der gesuchte Coefficient.

Ich muss zuerst zeigen, dass diese Definition nicht etwa den Widerspruch in sich enthält, von der Ausschliessung des untern Zeigers 0 abzuhängen. 
Es sei $n=6$, so wird $\left(\begin{array}{l}4 \\ 1\end{array}\right)\left(\begin{array}{l}5 \\ 2\end{array}\right)\left(\begin{array}{l}0 \\ 3\end{array}\right)\left(\begin{array}{l}0 \\ 4\end{array}\right)\left(\begin{array}{l}1 \\ 5\end{array}\right)\left(\begin{array}{l}1 \\ 6\end{array}\right)$ als einzelner Term im Ausdruck des Coefficienten von $x_{0} x_{1}^{2} x_{4} x_{5}$ vorkommen. Wählen wir 023456 als feste Reihe der untern Zeiger, so ist jener Term in der Form $\left(\begin{array}{l}4 \\ 0\end{array}\right)\left(\begin{array}{l}5 \\ 2\end{array}\right)\left(\begin{array}{l}0 \\ 3\end{array}\right)\left(\begin{array}{l}1 \\ 4\end{array}\right)\left(\begin{array}{l}1 \\ 5\end{array}\right)\left(\begin{array}{l}1 \\ 6\end{array}\right)$ darzustellen, indem man nur die Zeiger der Elemente $\left(\begin{array}{l}4 \\ 1\end{array}\right),\left(\begin{array}{l}0 \\ 4\end{array}\right)$ umkehrt (sie bilden eine offene Kette, die mit 1 beginnt, mit 0 schliesst und 4 zum verbindenden Zeiger hat), und diese neue Form desselben Terms entspricht wieder obiger Definition, indem, wenn unten 1 fehlt, oben $0^{1} 1^{3} 4^{1} 5^{1}$, d. i. 011145 zu permutiren ist. Soll 6 in der festen Reihe unterer Zeiger fehlen, so kehren wir zuerst $\left(\begin{array}{l}1 \\ 6\end{array}\right)$ in $\left(\begin{array}{l}6 \\ 1\end{array}\right)$ um, dann $\left(\begin{array}{l}4 \\ 1\end{array}\right)$ in $\left(\begin{array}{l}1 \\ 4\end{array}\right)$, dann $\left(\begin{array}{l}0 \\ 4\end{array}\right)$ in $\left(\begin{array}{l}4 \\ 0\end{array}\right)$ und

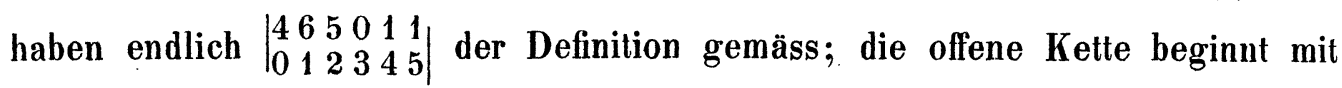
6 , endigt mit 0 und hat 1,4 als verbindende Zeiger. Wenn z. B. 1 als unterer Zeiger durch 0 ersetzt werden soll, so wird, da 0 in der obern Permutation nicht fehlt, und die übrigen Zeiger auch unten sich finden, stets eine offene Kette von Elementen da sein, die mit dem untern Zeiger 1 beginnt und mit dem obern 0 aufhört. Haben wir den zum Coefficienten von $x_{2} x_{3} x_{4} x_{5} x_{6}$ gehörenden Term $\left(\begin{array}{l}2 \\ 1\end{array}\right)\left(\begin{array}{l}3 \\ 2\end{array}\right)\left(\begin{array}{l}4 \\ 3\end{array}\right)\left(\begin{array}{l}5 \\ 4\end{array}\right)\left(\begin{array}{l}6 \\ 5\end{array}\right)\left(\begin{array}{l}0 \\ 6\end{array}\right)$ so darzustellen, dass 023456 als untere Zeigerreihe erscheint, so durchläuft die Kette alle sechs Elemente,

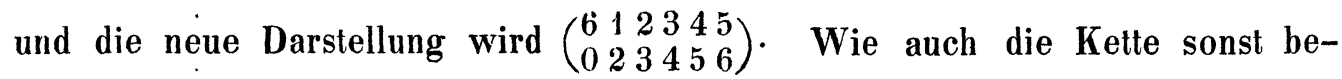
schaffen sein mag, durch die Umwendung aller ihrer Elemente wird der Wiederholungsexponent des heginnenden Zeigers oben um 1 vermehrt, derjenige des schliessenden oben um 1 vermindert, während alle verbindenden Zeiger in gleicher Anzahl oben bleiben, wie zuvor.

Wir denken uns nun das fragliche Polynom $(T)$ nicht nur nach den Coordinatencombinationen, sondern auch nach den Producten der Elemente in Terme aufgelöst und fragen nach dem Aggregate aller Terme, die den Factor $\left(\begin{array}{l}0 \\ 1\end{array}\right)$, aber nicht $x_{0}$ enthalten. Als feste Reihe der untern Zeiger sei $1234 \ldots n$ angenommen. Die Permutation der obern Zeiger wird dann mit 0 beginnen, aber sonst diesen Zeiger nicht enthalten. Damit nun die über $234 \ldots n$ stehende Permutation keinen Kreislauf erzeuge, so muss nothwendig 1 als ein der untern Reihe fremder Zeiger darin vorkommen, da der ebenfalls fremde Zeiger 0 schon ausgeschlossen ist. Folglich ist der Term auch noch durch $x_{1}$ theilbar. Sondern wir nun den Factor $\left(\begin{array}{l}0 \\ 1\end{array}\right) x_{1}$ ab, so bleibt ein Term, wie 
er nach der Definition der um einen Grad und eine Dimension niedrigeren Function $(T)$, die zu einem $V$ mit der Matrix $\left(\begin{array}{llll}1 & 2 & 3 & \ldots \\ 1 & 2 & 3 & \ldots\end{array}\right)$ gehörte, entspräche; und umgekehrt, wenn wir irgend einen Term dieser letzten Function $(\boldsymbol{T})$ mit $\left(\begin{array}{l}0 \\ 1\end{array}\right) x_{1}$ multipliciren, so haben wir einen Term der höheren Function $(T)$. Da dasselbe auch für die Factoren $\left(\begin{array}{l}0 \\ 2\end{array}\right) x_{2},\left(\begin{array}{l}0 \\ 3\end{array}\right) x_{3}, \ldots\left(\begin{array}{l}0 \\ n\end{array}\right) x_{n}$ bewiesen werden kann, so folgt, dass $(T)$ sich für $x_{0}=0$ auf das Product von $\left(\begin{array}{l}0 \\ 1\end{array}\right) x_{1}+\left(\begin{array}{l}0 \\ 2\end{array}\right) x_{2}+\cdots+\left(\begin{array}{l}0 \\ n\end{array}\right) x_{n}$ mit der erwähnten niedrigeren Function reducirt. Da endlich die Definition auch $\left(\begin{array}{l}0 \\ 1\end{array}\right)\left(\begin{array}{l}0 \\ 2\end{array}\right)\left(\begin{array}{l}0 \\ 3\end{array}\right) \ldots\left(\begin{array}{l}0 \\ n\end{array}\right) x_{0}^{n-1}$ als Anfangsterm von $(T)$ giebt, so ist die Identität der definirten Function $(\boldsymbol{T})$ mit der ursprünglichen $\boldsymbol{T}$ bewiesen.

Es bleibt noch übrig zu zeigen, dass die Anzahl der Terme des Coefficienten einer Coordinatencombination der Permutationszahl dieser letzten gleich ist. Setzen wir $V=\left(x_{0}+x_{1}+x_{2}+\cdots+x_{n}\right)^{2}$, so ist klar, dass alle Schnitte des Gebildes $x_{0} x_{1} x_{2} \ldots x_{n}=0$ mit dem polaren Gebilde in die lineare Gleichung $x_{0}+x_{1}+\cdots+x_{n}=0$ hinein fallen, und dass daher $T=\left(x_{0}+x_{1}+\cdots+x_{n}\right)^{n-1}$ wird. Da jedes Elementproduct jetzt $=1$ ist, so ist ferner klar, dass die Anzahl solcher in irgend einem Coefficienten dem Coefficienten der betreffenden $\mathrm{Co}_{0}$ ordinatencombination in der Entwicklung von $(\Sigma x)^{n-1}$ gleich ist. Man kann aber die Richtigkeit dieser Anzahl auch aus obiger Definition der Function $T$ herleiten, wenn man mit $x_{0}^{\alpha} x_{1}^{\beta},(\alpha+\beta=n-1)$, beginnt, dann $x_{1}^{\alpha} x_{2}^{\beta} x_{3}^{\gamma}$, $(\alpha+\beta+\gamma=n-1)$, betrachtet und so fortgeht, indem man immer den Satz für die niedrigere Stufe als schon bewiesen voraussetzt. Das Fundament der Beweisführung würde dann sein, dass in der Entwicklung von

$$
\left(1+\left(\begin{array}{l}
\alpha \\
1
\end{array}\right) x+\left(\begin{array}{c}
\alpha \\
2
\end{array}\right) x+\cdots\right)\left(1+\left(\begin{array}{c}
\beta \\
1
\end{array}\right) x+\left(\begin{array}{l}
\beta \\
2
\end{array}\right) x^{2}+\cdots\right)\left(1+\left(\begin{array}{l}
\gamma \\
1
\end{array}\right) x+\left(\begin{array}{l}
\gamma \\
2
\end{array}\right) x^{2}+\cdots\right) \times \cdots
$$

der Coefficient von $x^{r}$ gleich $\left(\begin{array}{c}\alpha+\beta+\gamma \\ r\end{array}\right)$ ist, wo $1+\left(\begin{array}{c}\alpha \\ 1\end{array}\right) x+\left(\begin{array}{l}\alpha \\ 2\end{array}\right) x^{2}+\cdots$ die Entwicklung von $(1+x)^{\alpha}$ bedeutet.

- Bern, 1865. 